\title{
T2K neutrino-nucleus cross-section results
}

\author{
M. Buizza Avanzini*广 \\ CNRS/IN2P3/LLR, Palaiseau, France \\ E-mail: buizza@in2p3.fr
}

\begin{abstract}
A detailed understanding of neutrino $(v)$-nucleus interactions is essential for the precise measurement of neutrino oscillations at long baseline experiments, such as T2K. Thanks to its near detector complex, the $\mathrm{T} 2 \mathrm{~K}$ experiment is providing a variety of neutrino cross-section measurements on different targets and at different neutrino energies. After an overview of the strategy adopted to reduce the model dependence, the most recent results of the T2K experiment are presented in this work.
\end{abstract}

Neutrino Oscillation Workshop (NOW2018)

9 - 16 September, 2018

Rosa Marina (Ostuni, Brindisi, Italy)

\footnotetext{
* Speaker.

${ }^{\dagger}$ on behalf of the $\mathrm{T} 2 \mathrm{~K}$ Collaboration
} 


\section{The T2K experiment}

The T2K (Tokai to Kamioka) experiment is a long-baseline neutrino oscillation experiment based in Japan [1]. The neutrino beam is produced by the J-PARC accelerator complex in Tokai, and travels toward the far detector Super-Kamiokande, $295 \mathrm{~km}$ from the neutrino production point and $2.5^{\circ}$ off-axis w.r.t. the beam direction. The main goal of $\mathrm{T} 2 \mathrm{~K}$ is the observation of the electron (anti-)neutrino appearance in a muon (anti-)neutrino beam.

A near detector complex is located $280 \mathrm{~m}$ from the beam target and is used to measure the unoscillated flux and the (anti-)neutrino cross-sections. The complex consists of two detectors. The first one, INGRID, is on the beam axis and it is mainly made of iron; also a water [2] and a plastic scintillator modules can be added. The second one, ND280, is on the same axis as SuperKamiokande and is made of several kind of targets, mainly water, hydrocarbon and lead.

\section{The T2K strategy for the cross-section measurements}

With the goal of providing useful information for a deeper understanding of the $v$-nucleus scattering without dependence on theoretical models, a detailed strategy for the cross-section extraction has been elaborated within the T2K collaboration.

The topology catalogue. Depending on their energy, neutrinos can interact with nucleons via charged-current $(\mathrm{CC})$ quasi elastic $(\mathrm{QE}), \mathrm{CC}$ resonant pion production (CCRES) or CC deep inelastic scattering (CCDIS). However, present and future long baseline experiments, use complex targets where nucleons are bounded in nucleus; $v$ interactions are thus affected by nuclear effects that can alter the final state particle types and kinematics. Moreover, correlations between bounded nucleons can lead to multinucleon knock-out (2p2h). Finally, final state hadrons can scatter or be re-absorbed in the nuclear environment, via the so called final state interactions (FSI), which can also produce additional final state particles. Indeed, nuclear effects, combined with detector limited acceptance and efficiencies, make it very hard to recognize the true $v$ interaction. In T2K, the signal definition is, thus, based on the final state topology without any attempt to infer about the true $v$ interactions, a procedure that would be highly model dependent.

Multi-flux and multi target measurements. Thanks to the off-axis technique, the T2K flux at ND280 is peaked $\sim 0.6 \mathrm{GeV}$, where $v$ mainly interact via CCQE; on the other hand, at the on-axis detector, the neutrino energy is peaked at $\sim 1 \mathrm{GeV}$ where CCRES and CCDIS interactions are important. All that allows us to explore a wide set of interaction channels. Moreover, as previously described, various targets can be studied, namely water, hydrocarbon, iron and lead.

Observables and techniques. In order to further limit the model dependence, T2K cross-section measurements are usually performed in terms of the observables effectively measured in the detectors, namely muon kinematics, and in some cases hadron kinematics (protons or pions over threshold). When possible, double differential cross-sections are delivered, thus reducing the model dependence related to the efficiency corrections. A data-driven regularization of the unfolded results is encouraged and forward-folding techniques are currently being studied.

\section{T2K near detectors cross-section results}

With $\sim 15$ publications and additional $\sim 20$ analyses currently ongoing, the T2K collaboration 
is very productive in the field of neutrino cross-section measurements.

CC inclusive channel. The most recent paper [3] reports a $4 \pi$ double differential measurement of the CC inclusive channel for $v_{\mu}$ at ND280. The unfolded cross-sections are extracted via a binned likelihood fitter, using alternatively GENIE [4] and NEUT [5] as prior, thus explicitly cross checking the independence of the results from the prediction. Comparing, then, extracted results with GENIE and NEUT doesn't show preference for a particular generator. Concerning the onaxis detectors, a paper reporting the $v_{\mu}$ cross-section measured on water, hydrocarbon and iron is in preparation. In left panel of Fig. 1 we report the three absolute cross-sections and their ratios; a the reduction of the systematics errors (detector and flux related) is evident. This kind of measurements allow to probe A-scaling models, describing how cross-sections scale with the nucleus size.
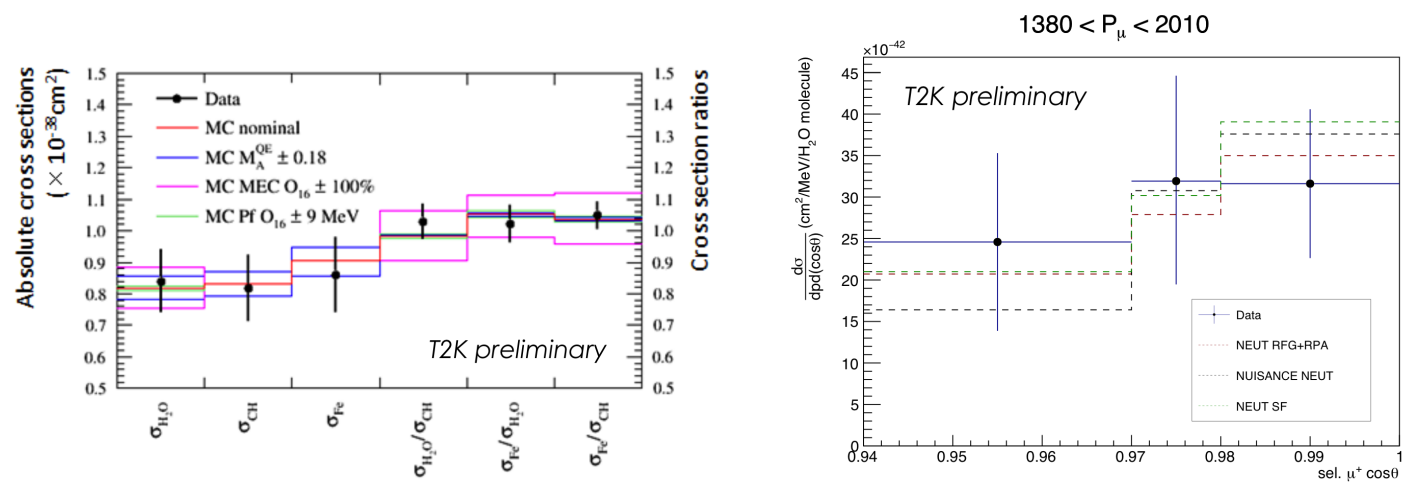

Figure 1: Left: CC-inclusive cross-sections on water, hydrocarbon and iron and their ratios, performed with INGRID and the Proton and Water modules for $\theta_{\mu}<45^{\circ}$ and $p_{\mu}>0.4 \mathrm{GeV}$. Right: $\bar{v}_{\mu}$ CC0 $\pi$ double differential cross-section on water, for a sub-set bins, compared with different NEUT predictions.

CCO $\pi$ channel. The CC channel without pion in the final state is the most relevant at T2K off-axis energies, since it is mainly composed of CCQE interactions. A deep study of this channel is thus fundamental for the T2K oscillation analysis. Several measurements have been provided by T2K, in particular: the off-axis $v_{\mu} \mathrm{CC} 0 \pi$ on hydro-carbon [6] and the first $\mathrm{CC} 0 \pi$ measurement on water [7]. Both are double differential measurements in muon kinematics and, compared with standard neutrino generator predictions, show a preference for models including multi-nucleon ejection. In order to better explore multi nucleon effects, three analyses looking at the $\mathrm{CC} 0 \pi$ channel plus $\mathrm{N}$ protons in the final state have been performed and recently published [8]. They represent a powerful tool to probe nuclear effects by looking at the proton kinematics and possible combinations with the muon kinematics (proton inferred kinematics and single transverse variables). Finally, T2K has provided the first CC $0 \pi$ cross-section measurement of $\bar{v}_{\mu}$ on water. The double differential crosssection is extracted as a function of the muon kinematics and in right panel of Fig. 1 we report a comparison of the preliminary unfolded results w.r.t. different NEUT predictions.

CC1 $\pi$ channel. Subdominant for T2K, but very relevant for NOvA [9] and DUNE [10], the CC channel with emission of one charged pion (mainly CCRES interactions) is also studied and many 

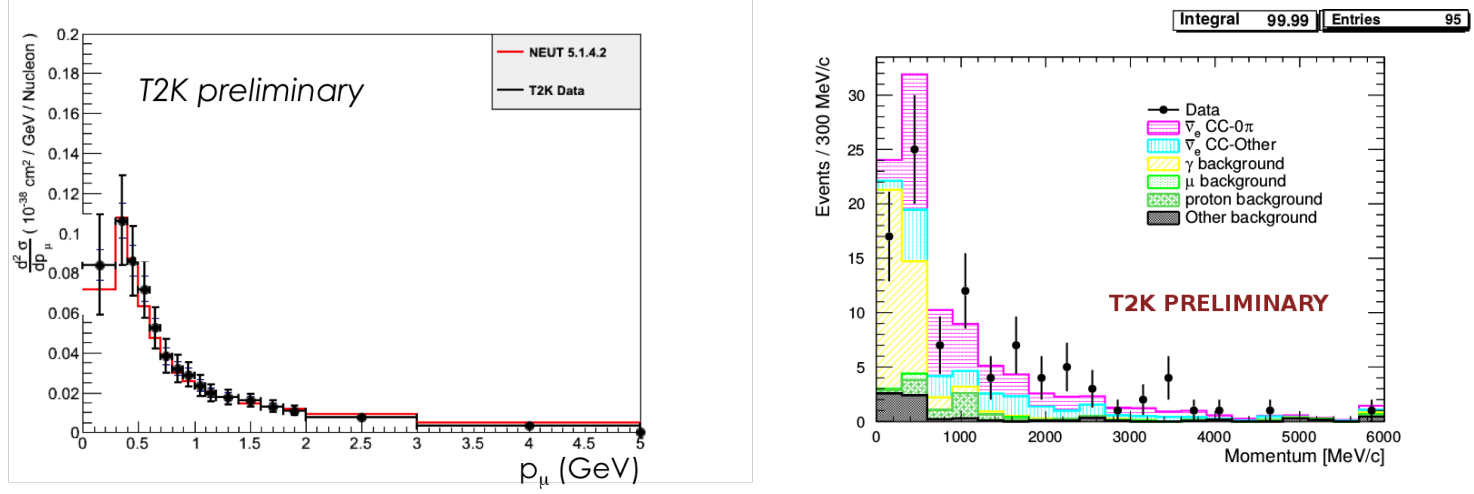

Figure 2: Left: differential $\mathrm{CC} 1 \pi^{+}$cross-section measurements on hydrocarbon at ND280 as a function of the muon momentum for $\cos \theta_{\mu}>0.2$ and $p_{\mu}>0.2 \mathrm{GeV}$, compared with NEUT prediction. Right: $\bar{v}_{e}$ (magenta) selection in the $\bar{v}_{\mu}$ beam; the main background is represented by $\gamma$ 's (yellow).

analyses are currently on going. After the first measurement of the $v_{\mu} \mathrm{CC} 1 \pi^{+}$single differential cross-section on water as a function of muon and pion kinematics [11], a paper reporting the $v_{\mu}$ $\mathrm{CC} 1 \pi^{+}$cross-section on carbon in a large number of variables is currently in preparation. In left panel of Fig. 2, we report preliminary results of differential $\mathrm{CC} 1 \pi^{+}$cross-section measurements on hydrocarbon at ND280 as a function of the muon momentum.

Rare events measurements. Also more challenging analyses are developed at T2K. This is the case of the single $\pi^{0}$ production rate in neutral current $v$ interactions on water [12]; $\pi^{0}$ are, indeed, a feared source of background for the $v_{e}$ appearance channel in Super-Kamiokande. Another dangerous background for the appearance channel is represented by the intrinsic (anti-) $v_{e}$ contamination in the (anti-) $v_{\mu}$ beam; thus the measurement of the (anti-) $v_{e}$ cross-section is extremely important. This analysis is extremely challenging due to the relative low (anti-) $v_{e}$ statistics at the near detector and the high $\gamma$ background. In right panel of Fig. 2, we report the preliminary results of the $\bar{v}_{e}$ selection in the $\bar{v}_{\mu}$ beam performed with ND280.

\section{Summary and outlook}

The T2K collaboration has provided a wide set of measurements of $v$-nucleus cross-sections, and keeps on exploiting current data and detectors to further improve the knowledge in this fundamental field. New exciting results are expected in incoming years thanks to a new near detector, WAGASCI [2], just installed at JPARC, and to the upgrade of ND280 [13], expected by 2021.

\section{References}

[1] K. Abe et al., Nucl. Instrum. Meth. A 659, 106 (2011).

[2] T. Ovsiannikova et al., J. Phys. Conf. Ser. 675 no.1, 012030 (2016).

[3] K. Abe et al., Phys. Rev. D 98, 012004 (2018).

[4] C. Andreopoulos et al., arXiv:1510.05494. 
[5] Y. Hayato, Acta Phys. Polon. B 40, 2477 (2009).

[6] K. Abe et al., Phys. Rev. D 93, 112012 (2016).

[7] K. Abe et al., Phys. Rev. D 97, 012001 (2018).

[8] K. Abe et al., Phys. Rev. D 98, 032003 (2018).

[9] P. Adamson et al., Phys. Rev. Lett. 116, 151806 (2016).

[10] R. Acciarri et al., arXiv:1601.05471.

[11] K. Abe et al., Phys. Rev. D 95, 012010 (2017).

[12] K. Abe et al., Phys. Rev. D 97, 032002 (2018).

[13] A. Blondel et al., CERN-SPSC-2018-001. SPSC-P-357 (2018). 